\title{
Fatores associados ao abandono do tratamento da tuberculose: estudo de casos e controles
}

\section{Factors associated with abandonment of tuberculosis treatment: a case-control study}

\author{
Karla Poersch ${ }^{1}$ (D), Juvenal Soares Dias da Costa $^{1}$ (D) \\ 'Programa de Pós-graduação em Saúde Coletiva, Universidade do Vale do Rio dos Sinos (UNISINOS) - São Leopoldo (RS), Brasil.
}

\begin{abstract}
Como citar: Poersch K, Dias-da-Costa JS. Fatores associados ao abandono do tratamento da tuberculose: estudo de casos e controles. Cad Saúde Colet, 2021;29(4):485-495. https://doi.org/10.1590/1414-462X202129040
\end{abstract}

\begin{abstract}
Resumo
Introdução: $\mathrm{O}$ abandono do tratamento da tuberculose (TB) está associado à manutenção da doença, resistência medicamentosa e morbimortalidade. Objetivo: Avaliar a tendência da TB e identificar os fatores de risco associados ao abandono do tratamento no município de Sapucaia do Sul, no Rio Grande do Sul, nos anos de 2006 a 2012. Método: Estudo de casos e controles com dados secundários notificados no Sistema de Informação. Os casos foram de pacientes que abandonaram o tratamento. Como controles, foram sorteadas pessoas que completaram o tratamento e obtiveram cura. O pareamento foi realizado de acordo com a data do diagnóstico da doença. Foram incluídos quatro controles para cada caso. Resultados: Foram notificados 660 casos de TB no período. O abandono manteve-se acima dos 5\%, com aumento a partir de 2009. Na análise ajustada, idade de 16 a 29 anos (OR = 16,9; IC95\%: 3,78-76,0; $\mathrm{p}<0,001$ ), idade de 30 a 49 anos (OR =9,9; IC95\%: 2,25-43,8; $<<0,001)$, entrada como recidiva após cura (OR = 2,83; IC95\%: 1,30-6,14; $p<0,01)$ e drogadição (OR = 3,94; IC95\%: 1,13-13,6; $p=0,03$ ) permaneceram associadas com o abandono do tratamento. Conclusão: Indivíduos jovens, que já haviam apresentado a doença e se curado e que faziam o uso de drogas ilícitas, abandonaram o tratamento.
\end{abstract}

Palavras-chave: tuberculose; pacientes desistentes do tratamento; estudos de casos e controles.

\begin{abstract}
Background: The abandonment of tuberculosis (TB) treatment is associated with maintenance of the disease, drug resistance, and morbidity and mortality. Objective: To evaluate the tendency of TB and identify the risk factors associated with the abandonment of treatment in the city of Sapucaia do Sul in Rio Grande do Sul state, from 2006 to 2012. Method: This is a case-control study carried out with secondary data reported in the Information System. The case study had the patients who abandoned treatment. The control study had individuals that completed the treatment and were cured. The pairing was performed according to the date of diagnosis of the disease. Four controls were included for each case. Results: 660 cases of TB were reported in the period. The abandonment remained above $5 \%$, with an increase from 2009 on. In the adjusted analysis, age 16 to 29 years (OR = 16.9; 95\% Cl: $3.78-76.0$; $\mathrm{p}<0.001)$, age 30 to 49 years (OR $=9.9 ; 95 \% \mathrm{Cl}: 2.25-43.8 ; \mathrm{p}<0.001)$, type of entry as relapse after cure $(\mathrm{OR}=2.83 ; 95 \% \mathrm{Cl}: 1.30-6.14 ; \mathrm{p}<0.01)$ and drug addiction $(\mathrm{OR}=3.94 ; 95 \% \mathrm{Cl}: 1.13-13.6 ; \mathrm{p}=0.03)$ remained associated with abandonment of treatment. Conclusion: Young individuals, who had already presented the disease and cured, who used illicit drugs, were those who abandoned treatment.
\end{abstract}

Keywords: tuberculosis; patient dropouts; case-control studies.

Trabalho realizado na Instituição UNISINOS- São Leopoldo (RS), Brasil

Correspondência: Karla Poersch. E-mail: karlapoersch@yahoo.com.br

Fonte de financiamento: $\mathrm{O}$ estudo não contou com nenhum tipo de financiamento. Foi aprovado pelo CEP Unisinos parecer $n^{\circ} 15 / 031$.

Conflito de interesses: declaramos que não há conflito de interesse e que os autores contemplam todos os critérios de autoria. Recebido em: Jul. 03, 2019. Aprovado em: Set. 02, 2020 


\section{INTRODUÇÃO}

A tuberculose (TB) é uma doença transmissível, considerada um importante problema de saúde, sendo uma das dez principais causas de morte em todo o mundo. Apesar de ser uma doença que pode ser prevenida e curada, ainda é altamente prevalente em condições sociais de vulnerabilidade, além de contribuir para a manutenção da desigualdade social'1.

Considerada uma doença negligenciada por sua alta prevalência em países em desenvolvimento, está associada com elevada proporção de morbidade e mortalidade no Brasil2 , onde, no ano de 2018, foram registrados 72.788 casos novos de TB e, em 2017, ocorreram 4,5 mil óbitos relacionados à doença, representando um coeficiente de mortalidade de 2,2 óbitos/100.000 habitantes ${ }^{3}$.

Diante desse panorama, o Ministério da Saúde do Brasil vem desenvolvendo ações prioritárias para o controle da doença por meio do Programa Nacional de Controle da Tuberculose (PNCT). As ações envolvem distribuição de medicamentos, cuidado na atenção primária à saúde, esquemas padronizados de tratamento, busca de sintomáticos respiratórios, manutenção de sistema de informação efetivo e adoção do tratamento diretamente observado (TDO), que consiste na tomada supervisionada da medicação4.

Em 2017, foi lançado o Plano Nacional pelo Fim da Tuberculose como Problema de Saúde Pública, com recomendações específicas considerando o contexto socioeconômico e epidemiológico dos municípios, com foco em três pilares: prevenção e cuidado integrado e centrado no paciente; políticas arrojadas e sistema de apoio; e intensificação da pesquisa e inovação $0^{5,6}$.

No Brasil, o esquema de tratamento da TB é padronizado, com duração de seis meses, disponibilizado gratuitamente pelo Sistema Único de Saúde (SUS), mas a efetividade do tratamento varia muito, estando em torno de $70 \%$ na média nacional ${ }^{7}$. A rápida melhora dos sintomas pela redução da carga bacilar leva os pacientes a acreditar que estão curados, o que contribui para o abandono e o surgimento de formas resistentes do Mycobacterium tuberculosis ${ }^{8,9}$.

Fatores socioeconômicos da população afetada e a qualidade dos serviços de saúde também são apontados como barreiras para o sucesso do tratamento da TB, sendo o abandono do tratamento uma das principais limitações para o combate e a cura da TB, pois, além de aumentar os custos do tratamento, contribui para elevar as taxas de mortalidade e de recidiva da doença, além de facilitar a seleção de bacilos resistentes ${ }^{10-14}$.

Para o PNCT, diversos municípios brasileiros são considerados prioritários por apresentarem elevada incidência de casos, bem como elevado número de abandonos do tratamento. No Rio Grande do Sul (RS), em 2014, a incidência de TB foi de 40,7 casos por 100.000 habitantes, 0 coeficiente de mortalidade foi de 2,3/100.000 habitantes e 20 municípios foram considerados prioritários por possuir o maior número de $\operatorname{casos}^{15,16}$. Sapucaia do Sul, município considerado prioritário, tinha em 2005 taxa bruta de prevalência de 67,9 casos por 100.000 habitantes $(66,5-69,3)$, sendo a taxa bruta de incidência de 59,7 casos por 100.000 habitantes $(58,4-61,0)$ e taxa de abandono de $11,2 \%$ nesse período ${ }^{17}$. Com dados muito superiores às taxas estadual e nacional, implantou naquele mesmo ano o Programa Municipal de Controle da Tuberculose (PMCT), visando melhorar o acesso aos serviços de saúde e promover a adesão ao tratamento, introduzindo a estratégia de TDO e a descentralização das ações ${ }^{18}$.

Mesmo diante dos esforços realizados, estudo realizado no município entre 2000 e 2008 demonstrou que os indicadores ainda eram insatisfatórios em relação às metas para o controle da doença, a incidência de TB estava elevada e a taxa de abandono do tratamento se mantinha acima da proposta pelo $\mathrm{PNCT}^{19}$. Sendo assim, este estudo teve como objetivo avaliar a tendência da TB no município após a implantação do Programa, bem como identificar os fatores de risco associados ao abandono do tratamento da TB no município de Sapucaia do Sul (RS), entre os anos de 2006 a 2012. 


\section{MÉTODO}

Foi realizado estudo de casos e controles com base em dados secundários notificados no Sistema de Informação de Agravos de Notificação da Secretaria Municipal de Saúde (SINAN/ SMS) de Sapucaia do Sul (RS) no período de 2006 a 2012.

Sapucaia do Sul está localizada na Região Metropolitana de Porto Alegre. De acordo com os dados do Instituto Brasileiro de Geografia e Estatística (IBGE), em 2010 a população residente no município era de 130.957 habitantes (apenas 488 na zona rural), distribuídos em $58,309 \mathrm{~km}^{2}$, sendo a densidade demográfica do município de $2.245,9 \mathrm{hab} / \mathrm{km}^{2}$. O Índice de Desenvolvimento Humano (IDH) do município nesse mesmo período foi de 0,726 , apresentando um elevado nível de desigualdade social, medido pelo índice de Gini de 0,41. Entre outros indicadores que confirmavam a vulnerabilidade do município, o IBGE elencou também a taxa de incidência de pobreza, que, em Sapucaia do Sul, foi de 31,4\%, percentual bastante superior ao da capital $(23,7 \%)^{20}$. Em 2009, a rede de atenção primária à saúde apresentava 13 unidades de Estratégia Saúde da Família (ESF), 5 Unidades Básicas de Saúde (UBS) e 1 Unidade Móvel de Atendimento. $\mathrm{O}$ sistema de saúde ainda se complementava por unidades de atenção secundária, incluindo serviço de pronto-atendimento, central de especialidades - local de atendimento dos casos de TB -, ambulatório de infectologia, clínica para assistência à saúde da mulher e dois serviços de atendimento em saúde mental. A atenção terciária dispunha de hospital que atendia emergências, internações e casos que necessitavam de UTI. Toda a rede municipal de saúde estava capacitada para atuar no PMCT.

No presente estudo, foram incluídos todos os pacientes classificados como eventos novos de TB ou recidivas após cura, notificados no SINAN/SMS e residentes no município. Foram excluídos outros tipos de entrada (reingresso após abandono, transferência e entrada ignorada), bem como pacientes menores de 15 anos de idade, uma vez que a administração da medicação poderia estar relacionada à supervisão dos pais e/ou responsáveis.

Os casos foram definidos como os pacientes que abandonaram o tratamento, ou seja, aqueles que deixaram de comparecer à unidade de saúde por mais de 60 dias após a última consulta, ficando sem a medicação por pelo menos 30 dias. Como controles, foram sorteadas pessoas que completaram o tratamento, obtiveram cura e que tiveram uma evolução favorável. O pareamento foi realizado de acordo com a proximidade da data do diagnóstico da doença e/ou data inicial do tratamento (até 15 dias antes ou depois). Foram incluídos quatro controles para cada caso.

A amostra foi estimada a partir de nível de confiança de $90 \%$, poder de $80 \%$, proporção de 4 controles para 1 caso, frequências de exposições no grupo não doente de até 7\%, com razão de odds de 3,0, sendo necessários 315 indivíduos, ou seja, 63 casos e 252 controles.

Da ficha de notificação, foram coletados: data de notificação e dados sociodemográficos, como sexo, idade, raça/cor, escolaridade e se era institucionalizado. Quanto aos aspectos relacionados ao diagnóstico da TB, foram coletados: tipo de entrada (caso novo ou recidiva após cura), forma de TB (pulmonar, extrapulmonar ou pulmonar + extrapulmonar), baciloscopia de escarro (positiva, negativa e não realizada) e local de diagnóstico (atenção primária, secundária ou terciária). Em relação às características clínicas dos pacientes, foram coletados alguns agravos associados, como infecção por HIV, drogadição, diabete mellitus (DM), doença mental, tabagismo e alcoolismo. As variáveis de acompanhamento da TB foram necessidade de TDO (sim ou não), contatos identificados e contatos examinados.

$\mathrm{Na}$ análise, inicialmente foram descritas as taxas de incidência ( $\mathrm{n}^{\circ}$ de casos de TB por ano/população do município por ano), a coinfecção HIV/TB e as taxas de abandono entre 2006 e 2012. Posteriormente, foram realizadas a descrição e a comparação dos casos e dos controles quanto às características sociodemográficas, aspectos relacionados ao diagnóstico, características clínicas dos pacientes e de acompanhamento da doença. Por meio do Programa Stata 13, foi feita a análise bruta mediante razões de odds, além de seus intervalos de confiança a $95 \%$ e testes estatísticos para identificar os fatores de risco associados ao abandono do tratamento da TB. Foi realizada análise ajustada mediante regressão logística condicional seguindo o modelo hierarquizado. Ingressaram no modelo as variáveis cujo $p$-valor foi $\leq 0,05$. No primeiro nível do modelo hierarquizado estavam as variáveis sociodemográficas. O segundo nível foi constituído pelos aspectos relacionados ao diagnóstico. No terceiro nível estavam as variáveis de características clínicas dos pacientes e de acompanhamento da doença. Todas as variáveis determinavam o desfecho. 
O Projeto foi aprovado pelo Comitê de Ética em Pesquisa da Universidade do Vale do Rio dos Sinos (UNISINOS) mediante Parecer no 15/031, emitido em 10 de abril de 2015.

\section{RESULTADOS}

Durante os anos de 2006 a 2012, foram registrados no SINAN 660 casos de TB no município de Sapucaia do Sul, com aumento expressivo do número de casos após 2009. A taxa de incidência de TB apresentou grande variabilidade, passando de 59,6 em 2006 para 93,5 casos por 100.000 habitantes em 2011, alcançando 87,7 casos por 100.000 habitantes em 2012. Também foi constatada elevação nas taxas de coinfecção HIV/TB, principalmente nos últimos dois anos. Em relação ao abandono do tratamento, o percentual manteve-se acima dos 5\% em seis anos, observando-se aumento a partir de 2009 (Tabela 1).

Tabela 1. Número de casos, taxa de incidência de tuberculose, taxa de incidência de coinfecção HIV/TB e percentual de abandono de tratamento. Sapucaia do Sul, Rio Grande do Sul, 2006-2012

\begin{tabular}{lccccccc} 
& $\mathbf{2 0 0 6}$ & $\mathbf{2 0 0 7}$ & $\mathbf{2 0 0 8}$ & $\mathbf{2 0 0 9}$ & $\mathbf{2 0 1 0}$ & $\mathbf{2 0 1 1}$ & $\mathbf{2 0 1 2}$ \\
\hline Número de casos & 81 & 73 & 86 & 71 & 110 & 123 & 116 \\
\hline Taxa incidência TB/100.000 & 59,6 & 52,9 & 68,2 & 56,2 & 84,0 & 93,5 & 87,7 \\
\hline Taxa incidência coinfecção HIV/TB/100.000 & 10,3 & 15,9 & 13,5 & 7,9 & 13,7 & 28,9 & 21,2 \\
\hline \% abandono & 6,2 & 9,6 & 4,7 & 14,1 & 10,9 & 9,8 & 11,2 \\
\hline
\end{tabular}

Na comparação das distribuições entre casos e controles quanto às características sociodemográficas e em relação aos aspectos relacionados ao diagnóstico de TB, foi constatada maior ocorrência de casos nas faixas de 16 a 29 anos $(p=0,001)$, naqueles com tipo de entrada classificado como recidiva após cura $(p=0,003)$, com baciloscopia de escarro positiva $(p=0,03)$ e com diagnóstico realizado na atenção terciária $(p=0,03)$. Não foram encontradas diferenças na distribuição em relação ao sexo, raça/cor, escolaridade, institucionalização e forma de TB (Tabela 2).

Entre as características clínicas dos pacientes, foi encontrada maior ocorrência de casos nos indivíduos com HIV positivo $(p=0,02)$ e drogadição $(p<0,0001)$. Não foram observadas diferenças na distribuição de casos e de controles quanto à presença de diabete mellitus, ocorrência de doença mental, tabagismo e alcoolismo. Em relação ao acompanhamento da TB, o TDO não se mostrou diferente entre os grupos. O número de contatos identificados foi significativamente superior no grupo controle $(p=0,007)$. Os contatos foram mais examinados entre os controles, entretanto apresentaram significância limítrofe $(p=0,05)$ (Tabela 2).

$\mathrm{Na}$ análise bruta por regressão logística, idade entre 16 e 29 anos (OR = 18,8; IC95\%: 4,20$84,3 ; p=0,0001)$, cor não branca ( $O R=1,93 ; I C 95 \%: 1,02-3,64 ; p=0,04)$, tipo de entrada como recidiva após cura ( $O R=2,7 ; \mathrm{IC} 95 \%: 1,38-5,30 ; \mathrm{p}=0,004)$ e diagnóstico na atenção terciária $(\mathrm{OR}=1,5$; IC95\%: 0,66-3,35; $\mathrm{p}=0,02)$ mostraram-se como fatores de risco para o abandono do tratamento da TB. Entre as características clínicas dos pacientes que estiveram associadas ao abandono, tiveram destaque a infecção por HIV (OR =3,44; IC95\%: 1,73-6,85; $p=0,001)$ e a drogadição ( $O R=9,15 ; \mathrm{IC} 95 \%: 3,80-22,0 ; p<0,0001)$. Não ter contatos identificados ( $O R=3,98$; IC95\%: 1,75-9,00; $p=0,001$ ) nem contatos examinados (OR = 2,97; IC95\%: 1,10-8,00; $p=0,03$ ) também se mostraram como fatores de risco para o abandono do tratamento da TB (Tabela 3).

$\mathrm{Na}$ análise ajustada, no primeiro nível, correspondente às variáveis sociodemográficas, foi observada maior ocorrência de abandono nas faixas etárias de 16 a 29 anos e 30 a 49 anos. No segundo nível, os aspectos relacionados ao diagnóstico de TB foram ajustados por idade e foi verificado que o tipo de entrada como recidiva após cura estava associado com o abandono do tratamento $(p<0,01)$. No terceiro nível, características clínicas dos pacientes e de acompanhamento da TB foram ajustadas para idade e tipo de entrada como recidiva após cura e foi constatado que drogadição estava associada com o abandono do tratamento ( $p=$ 0,03 ). Portanto, no modelo final permaneceram associados ao abandono do tratamento da TB a idade, o tipo de entrada como recidiva após cura e a drogadição (Tabela 4). 
Tabela 2. Distribuição de casos e controles em relação às características sociodemográficas, aspectos relacionados ao diagnóstico, características clínicas e de acompanhamento da tuberculose. Sapucaia do Sul, Rio Grande do Sul, 2006-2012

\begin{tabular}{|c|c|c|c|c|c|}
\hline \multirow{2}{*}{ Variável } & \multicolumn{2}{|c|}{ Casos $(n=63)$} & \multicolumn{2}{|c|}{ Controles $(n=252)$} & \multirow{2}{*}{ p-valor } \\
\hline & $\mathbf{n}$ & $\%$ & $\mathbf{n}$ & $\%$ & \\
\hline \multicolumn{6}{|l|}{ Sexo } \\
\hline Feminino & 16 & 25,4 & 67 & 26,6 & \multirow{2}{*}{0,85} \\
\hline Masculino & 47 & 74,6 & 185 & 73,4 & \\
\hline \multicolumn{6}{|l|}{ Idade } \\
\hline 50 anos ou mais & 2 & 3,2 & 73 & 29 & \multirow{3}{*}{$0,001^{*}$} \\
\hline 30 a 49 anos & 32 & 50,8 & 118 & 46,8 & \\
\hline 16 a 29 anos & 29 & 46 & 61 & 24,2 & \\
\hline \multicolumn{6}{|l|}{ Raça/cor } \\
\hline Branca & 46 & 73 & 209 & 82,9 & \multirow{3}{*}{0,68} \\
\hline Não branca & 17 & 27 & 39 & 15,5 & \\
\hline Ignorada & 0 & - & 4 & 1,6 & \\
\hline \multicolumn{6}{|l|}{ Escolaridade } \\
\hline 12 anos ou mais & 5 & 7,9 & 24 & 9,5 & \multirow{5}{*}{0,93} \\
\hline 9 a 11 anos & 11 & 17,5 & 41 & 16,3 & \\
\hline 5 a 8 anos & 23 & 36,5 & 79 & 31,3 & \\
\hline 0 a 4 anos & 10 & 15,9 & 46 & 18,3 & \\
\hline Ignorada & 14 & 22,2 & 62 & 24,6 & \\
\hline \multicolumn{6}{|l|}{ Institucionalizado } \\
\hline Sim & 4 & 6,3 & 12 & 4,8 & \multirow{3}{*}{0,88} \\
\hline Não & 52 & 82,5 & 212 & 84,1 & \\
\hline Ignorado & 7 & 11,1 & 28 & 11,1 & \\
\hline \multicolumn{6}{|l|}{ Tipo de entrada } \\
\hline Caso novo & 46 & 73 & 222 & 88,1 & \multirow{2}{*}{$0,003^{*}$} \\
\hline Recidiva após cura & 17 & 27 & 30 & 11,9 & \\
\hline \multicolumn{6}{|l|}{ Forma TB } \\
\hline Pulmonar & 56 & 88,9 & 214 & 84,9 & \multirow{3}{*}{0,17} \\
\hline Extrapulmonar & 4 & 6,3 & 33 & 13,1 & \\
\hline Pulmonar + extrapulmonar & 3 & 4,8 & 5 & 2 & \\
\hline \multicolumn{6}{|l|}{ Baciloscopia de escarro } \\
\hline Positiva & 52 & 82,5 & 165 & 65,5 & \multirow{3}{*}{$0,03^{*}$} \\
\hline Negativa & 8 & 12,7 & 54 & 21,4 & \\
\hline Não realizada & 3 & 4,8 & 33 & 13,1 & \\
\hline \multicolumn{6}{|l|}{ Local de diagnóstico } \\
\hline Atenção primária & 15 & 23,8 & 49 & 19,4 & \multirow{3}{*}{$0,03^{*}$} \\
\hline Atenção secundária & 31 & 49,2 & 166 & 65,9 & \\
\hline Atenção terciária & 17 & 27 & 37 & 14,7 & \\
\hline
\end{tabular}


Tabela 3. Análise bruta de abandono do tratamento da tuberculose em relação às características sociodemográficas, aspectos relacionados ao diagnóstico, características clínicas e de acompanhamento. Sapucaia do Sul, Rio Grande do Sul, 2006-2012

\begin{tabular}{|c|c|c|c|c|c|}
\hline \multirow{2}{*}{ Variável } & Casos & \multirow{2}{*}{$\begin{array}{l}\text { Controles } \\
\text { n (\%) }\end{array}$} & \multirow{2}{*}{$\begin{array}{l}\text { Razão de } \\
\text { odds }\end{array}$} & \multirow{2}{*}{$\begin{array}{l}\text { Intervalo de } \\
\text { confiança }\end{array}$} & \multirow{2}{*}{ p-valor } \\
\hline & n (\%) & & & & \\
\hline \multicolumn{6}{|l|}{ Sexo } \\
\hline Feminino & $16(25,4)$ & $67(26,6)$ & 1 & & \multirow{2}{*}{0,85} \\
\hline Masculino & $47(74,6)$ & $185(73,4)$ & 1,06 & $0,57-1,97$ & \\
\hline \multicolumn{6}{|l|}{ Idade } \\
\hline 50 anos ou mais & $2(3,2)$ & $73(29,0)$ & 1 & & \multirow{3}{*}{$0,0001^{*}$} \\
\hline 30 a 49 anos & $32(50,8)$ & $118(46,8)$ & 10,6 & $2,40-46,7$ & \\
\hline 16 a 29 anos & $29(46,0)$ & $61(24,2)$ & 18,8 & $4,20-84,3$ & \\
\hline \multicolumn{6}{|l|}{ Raça/cor } \\
\hline Branca & $46(73)$ & $209(84,3)$ & 1 & & \multirow{2}{*}{$0,04^{*}$} \\
\hline Não branca & $17(27)$ & $39(15,7)$ & 1,93 & $1,02-3,64$ & \\
\hline \multicolumn{6}{|l|}{ Escolaridade } \\
\hline 12 anos ou mais & $5(7,9)$ & $24(9,5)$ & 1 & & \multirow{4}{*}{0,95} \\
\hline 9 a 11 anos & $11(17,5)$ & $41(16,3)$ & 0,9 & $0,20-3,85$ & \\
\hline 5 a 8 anos & $23(36,5)$ & $79(31,3)$ & 1,23 & $0,42-3,62$ & \\
\hline 0 a 4 anos & $10(15,9)$ & $46(18,3)$ & 0,94 & $0,28-3,23$ & \\
\hline \multicolumn{6}{|l|}{ Institucionalizado } \\
\hline Não & $52(82,5)$ & $212(84,1)$ & 1 & & \multirow{2}{*}{0,76} \\
\hline Sim & $4(6,3)$ & $12(4,8)$ & 1,11 & $0,55-2,28$ & \\
\hline \multicolumn{6}{|l|}{ Tipo de entrada } \\
\hline Caso novo & $46(73,0)$ & $222(88,1)$ & 1 & & \multirow{2}{*}{$0,004^{*}$} \\
\hline Recidiva & $17(27,0)$ & $30(11,9)$ & 2,7 & $1,38-5,30$ & \\
\hline \multicolumn{6}{|l|}{ Forma TB } \\
\hline Pulmonar & $56(88,9)$ & $214(84,9)$ & 1 & & \multirow{3}{*}{0,26} \\
\hline Extrapulmonar & $4(6,3)$ & $33(13,1)$ & 0,47 & $0,15-1,38$ & \\
\hline Pulmonar + extrapulmonar & $3(4,8)$ & $5(2,0)$ & 2,19 & $0,52-9,20$ & \\
\hline \multicolumn{6}{|l|}{ Baciloscopia de escarro } \\
\hline Positiva & $52(86,7)$ & $165(75,3)$ & 1 & & \multirow{2}{*}{0,66} \\
\hline Negativa & $8(13,3)$ & $54(24,7)$ & 0,47 & $0,21-1,05$ & \\
\hline \multicolumn{6}{|l|}{ Local de diagnóstico } \\
\hline Atenção primária & $15(23,8)$ & $49(19,4)$ & 1 & & \multirow{3}{*}{$0,02^{*}$} \\
\hline Atenção secundária & $31(49,2)$ & $166(65,9)$ & 0,52 & $0,25-1,10$ & \\
\hline Atenção terciária & $17(27,0)$ & $37(14,7)$ & 1,5 & $0,66-3,35$ & \\
\hline
\end{tabular}


Tabela 4. Análise ajustada de abandono do tratamento da tuberculose em relação às características sociodemográficas, aspectos relacionados ao diagnóstico, características clínicas e de acompanhamento. Sapucaia do Sul, Rio Grande do Sul, 2006-2012

\begin{tabular}{|c|c|c|c|}
\hline Variável & Razão de odds & Intervalo de confiança & p-valor \\
\hline \multicolumn{4}{|l|}{ Idade $^{1}$} \\
\hline 50 anos ou mais & 1 & & \multirow{3}{*}{$<0,001^{*}$} \\
\hline 30 a 49 anos & 9,9 & $2,25-43,8$ & \\
\hline 16 a 29 anos & 16,9 & $3,78-76,0$ & \\
\hline \multicolumn{4}{|l|}{ Raça/cor ${ }^{1}$} \\
\hline Branca & 1 & & \multirow{2}{*}{0,18} \\
\hline Não branca & 1,57 & $0,81-3,06$ & \\
\hline \multicolumn{4}{|l|}{ Tipo de entrada ${ }^{2}$} \\
\hline Caso novo & 1 & & \multirow{2}{*}{$<0,01^{*}$} \\
\hline Recidiva após cura & 2,83 & $1,30-6,14$ & \\
\hline \multicolumn{4}{|c|}{ Local de diagnóstico ${ }^{2}$} \\
\hline Atenção primária & 1 & & \multirow{3}{*}{0,06} \\
\hline Atenção secundária & 0,57 & $0,25-1,29$ & \\
\hline Atenção terciária & 1,6 & $0,67-3,80$ & \\
\hline \multicolumn{4}{|l|}{ HIV $^{3}$} \\
\hline Negativo & 1 & & \multirow{2}{*}{0,25} \\
\hline Positivo & 1,65 & $0,70-3,87$ & \\
\hline \multicolumn{4}{|l|}{ Drogadição $^{3}$} \\
\hline Não & 1 & & \multirow{2}{*}{$0,03^{*}$} \\
\hline Sim & 3,94 & $1,13-13,6$ & \\
\hline \multicolumn{4}{|c|}{ Contatos identificados $^{3}$} \\
\hline Sim & 1 & & \multirow{2}{*}{0,14} \\
\hline Não & 2,53 & $0,74-8,66$ & \\
\hline \multicolumn{4}{|c|}{ Contatos examinados ${ }^{3}$} \\
\hline Sim & 1 & & \multirow{2}{*}{0,12} \\
\hline Não & 2,94 & $0,74-11,6$ & \\
\hline
\end{tabular}

Teste de Wald; ${ }^{*} p<0,05 ;{ }^{1}$ Variáveis ajustadas entre si; ${ }^{2}$ Variáveis ajustadas para idade; ${ }^{3}$ Variáveis ajustadas para idade e tipo de entrada

\section{DISCUSSÃO}

O estudo mostrou que o aumento dos casos de TB foi acompanhado pelo crescimento do abandono do tratamento no período analisado Em Sapucaia do Sul, houve aumento da incidência de TB em conjunto com a elevação dos casos de coinfecção com HIV/TB, assim como em âmbito nacional, em que foi observada tendência crescente e positiva para a incidência de TB/HIV em diversos estados brasileiros. Em 2016, 8\% das pessoas vivendo com HIV apresentavam TB no Brasil, sendo que os indivíduos coinfectados por TB/HIV mostravam alta taxa de abandono $(11,6 \%)$ e óbito $(17,8 \%)^{21-23}$. 
Os resultados do presente estudo apontaram que fatores comportamentais, como idade inferior a 50 anos, tipo de entrada como recidiva após cura e drogadição, foram preditores para o abandono do tratamento dos casos de TB no município.

Dados semelhantes foram encontrados em uma revisão integrativa sobre fatores associados ao abandono de tratamento da TB realizada em 2011 em estados do Brasil e no Peru, que apontou que homens jovens e em idade produtiva, com baixa escolaridade, que faziam uso de drogas e haviam realizado tratamento prévio para TB, eram os que abandonavam o tratamento ${ }^{24}$. Em outra revisão realizada em 2017 em diferentes países, os autores também observaram que uso de drogas, idade, sexo masculino, baixa escolaridade e renda estiveram associados ao abandono de tratamento9.

Em relação à idade, foi possível observar que adultos jovens foram os que mais abandonaram o tratamento, associação documentada em estudo realizado previamente no município ${ }^{25}$. No Brasil, assim como em outros países, pesquisas têm revelado que o abandono do tratamento tem sido mais frequente em adultos jovens e em idade produtiva ${ }^{14,26,27}$, sendo a faixa etária acima de 50 anos um fator de proteção ao abandono ${ }^{28}$.

Em um estudo ecológico realizado em Salvador, Bahia, no período de 2006 a 2015, com 1.611 casos de reingresso após o abandono, os autores também identificaram que a maior parte dos pacientes que abandonaram o tratamento da TB tinha entre 15 e 49 anos $^{29}$. $\mathrm{O}$ desfecho associado à idade tem sido apontado como reflexo do número de adultos jovens acometidos pela TB e maior predisposição desse grupo ao consumo de álcool e drogas, sendo uma importante causa de ruptura do acompanhamento do paciente no serviço de saúde ${ }^{9,30}$.

A recidiva de TB foi confirmada quando o paciente tinha diagnóstico de TB por bacteriologia positiva (microscopia e/ou cultura) e apresentava história de TB anterior curada com medicamentos antituberculose. Um estudo de coorte e um estudo transversal, ambos realizados em municípios prioritários para o controle da TB, identificaram que indivíduos previamente curados apresentaram maior risco de desenvolver novamente a doença quando comparados à população geral, e as chances de recidiva e de abandonar o tratamento também foram maiores em adultos jovens, do sexo masculino, baixa escolaridade, dependentes de álcool ${ }^{31,32}$.

Em estudo transversal realizado no Espírito Santo entre os anos de 2002 a 2012, os autores identificaram que indivíduos com recidiva da doença tiveram razão de chance ajustada de 7,72 ( $p<0,001$; IC95\%: 4,24-4,05) para resistência aos fármacos antituberculose em relação aos indivíduos que não tinham história de tratamento anterior (casos novos), o que contribuiu para o difícil controle da doença e o abandono do tratamento ${ }^{33}$.

No entanto, na presente pesquisa, foi observada apenas associação com o uso de drogas ilícitas. A dependência de drogas ilegais foi demonstrada como fator independente para a não adesão ao tratamento, quando analisada a situação epidemiológica da TB nos países integrantes do Mercosul, estando associada ao aumento da taxa de abandono ${ }^{34}$.

Estudo ecológico de séries temporais realizado em todas as capitais brasileiras, no período de 2001 a 2015, identificou que a taxa média de abandono foi de 13,6\%, e o uso de drogas ilegais foi um dos fatores associados ao abandono ${ }^{35}$. Em outro estudo transversal, realizado no município de Belém, Pará, com pacientes que reingressaram no tratamento de TB após abandoná-lo, os autores observaram que, dentro dos hábitos de vida associados ao abandono do tratamento, o uso de drogas ilícitas foi o mais prevalente ${ }^{36}$.

Ao longo dos anos, o uso de drogas lícitas ou ilícitas tem sido apontado como fator preditivo para o abandono, pois promove dificuldades em manter a regularidade do tratamento, apontando para a necessidade de uma avaliação adequada do comportamento e implementação de políticas para o cuidado dos usuários que apresentam essa combinação de doenças, cuja prevalência está aumentando no Brasil ${ }^{37}$.

Outro aspecto apontado pela presente pesquisa se relacionou à taxa de coinfecção HIV/TB. Em Sapucaia do Sul, a taxa de coinfecção HIV/TB foi de 21,2 casos por 100.000 habitantes em 2012, e em outro município prioritário do Rio Grande do Sul, Porto Alegre, foi detectado que 20 a 30\% dos casos apresentavam coinfecção HIV/TB, valores elevados quando comparados aos valores nacionais, em que a taxa de coinfecção não ultrapassou $10 \%{ }^{38}$. 
Diante dos desafios envolvidos no controle da doença, como o elevado número de casos absolutos de TB, abandono do tratamento e TBMR (Tuberculose Multirresistente), foi introduzido oficialmente no Brasil, em 1997, oTDO, que consiste na observação da ingestão dos medicamentos por um profissional capacitado, visando à adesão ao tratamento ${ }^{7,15,39}$. Em Sapucaia do Sul, diante das dificuldades no controle da doença, em 2005 foi implantado o PMCT no município, introduzindo a estratégia do TDO e a descentralização das ações. No entanto, no presente estudo, o TDO não esteve associado à diminuição do abandono do tratamento da TB no município.

Um trabalho realizado em Porto Alegre, uma das capitais brasileiras com maior incidência de TB e baixa taxa de cura no tratamento, mostrou que, apesar de a estratégia do TDO ter sido adotada por vários serviços, os problemas relacionados à falta de tempo para a sua prática e a deficiência de recursos humanos, de materiais e de transporte dificultavam a operacionalização desse modelo ${ }^{40}$.

Estudos têm apontado que o TDO parece não ter sido efetivamente implantado nos municípios, uma vez que as dificuldades encontradas para a implantação da estratégia acabaram não impactando de forma significativa os índices de cura e abandono do tratamento, indicando a importância do compromisso intersetorial e a garantia de recursos para a concretização do controle da TB ${ }^{41,42}$.

Diante dos achados da presente pesquisa, é possível realizar algumas reflexões sobre o TDO em Sapucaia do Sul. OTDO parece ter sido sugerido para a grande maioria dos pacientes, independentemente de suas características e sem considerar a capacidade dos serviços de executar essa operação. Provavelmente, uma contribuição do presente estudo tenha sido indicar algumas características mais evidentes para a seleção dos indivíduos que exigem mais atenção.

Ainda assim, é preciso destacar como limitação: a ausência de alguns dados no sistema de informação, bem como os problemas de completude dessas informações, o que prejudicou o conhecimento do perfil dos pacientes que abandonaram o tratamento de TB no município. Mesmo com versões aprimoradas, as notificações no SINAN ainda apresentam problemas na qualidade dos dados, o que gera ausência de dados epidemiológicos e clínicos, seja pela dificuldade do profissional responsável na coleta, seja pelo volume expressivo de campos a serem preenchidos ${ }^{43}$.

Foi possível constatar que indivíduos jovens, que já haviam apresentado a doença anteriormente e se curado e que faziam uso de drogas ilícitas, foram os que abandonaram o tratamento. Com o objetivo de minimizar o risco de abandono nessa população, o TDO foi instituído no município de Sapucaia do Sul e vem sendo considerado um facilitador na conclusão do tratamento, por ser capaz de aproximar os serviços de saúde e a comunidade. No entanto, no presente estudo, sua implantação não garantiu resultados promissores quanto às metas estabelecidas para o controle da doença.

\section{REFERÊNCIAS}

1. World Health Organization. Global tuberculosis report 2019. Geneva:WHO; 2019.

2. Oliveira RG. Sentidos das doenças negligenciadas na agenda da Saúde Global: o lugar de populações e territórios. Cien Saude Colet. 2018;23(7):2291-302. http://dx.doi.org/10.1590/1413-81232018237.09042018. PMid:30020382.

3. Brasil. Ministério da Saúde. Secretaria de Vigilância em Saúde. Brasil Livre da Tuberculose: evolução dos cenários epidemiológicos e operacionais da doença. Boletim Epidemiológico. 2019;50(9):1-18.

4. Baumgarten A, Rech RS, Bulgarelli PT, Souza KR, Santos CM, Frichembruder K, et al. Ações para o controle da tuberculoseno Brasil: avaliação da atenção básica. Rev Bras Epidemiol. 2019;22:e190031. http://dx.doi. org/10.1590/1980-549720190031. PMid:31038612.

5. Brasil. Ministério da Saúde. Secretaria de Vigilância em Saúde. Departamento de Vigilância das Doenças Transmissíveis. Brasil Livre da Tuberculose: Plano nacional pelo fim da tuberculose como problema de saúde pública. Brasília: Ministério da Saúde; 2017.

6. Pelissari DM, Rocha MS, Bartholomay P, Sanchez MN, Duarte EC, Arakaki-Sanchez D, et al. Identifying socioeconomic, epidemiological and operational scenarios for tuberculosis control in Brazil: an ecological study. BMJ Open. 2018;8(6):e018545. http://dx.doi.org/10.1136/bmjopen-2017-018545. PMid:29880560. 
7. Rabahi MF, Silva JLR Jr, Ferreira ACG, Tannus-Silva DGS, Conde MB. Tuberculosis treatment. J Bras Pneumol. 2017;43(6):472-86. http://dx.doi.org/10.1590/s1806-37562016000000388. PMid:29340497.

8. Park CK, Shin HJ, Kim YI, Lim SC, Yoon JS, Kim YS, et al. Predictors of Default from Treatment for Tuberculosis: a Single Center Case-Control Study in Korea. J Korean Med Sci. 2016;31(2):254-60. http:// dx.doi.org/10.3346/jkms.2016.31.2.254. PMid:26839480.

9. Ferreira MRL, Bonfim RO, Siqueira TC, Orfão NH. Abandono do tratamento da tuberculose: uma revisão integrativa. Rev Enferm Contemp. 2018;7(1):63-71. http://dx.doi.org/10.17267/2317-3378rec.v7i1.1579.

10. Anduaga-Beramendi A, Maticorena-Quevedo J, Beas R, Chanamé-Baca DM, Veramendi M, WiegeringRospigliosi A, et al. Factores de riesgo para el abandono del tratamiento de tuberculosis pulmonar sensible en un establecimiento de salud de atención primaria, Lima, Perú. Acta Méd Peruana. 2016;33(1):21-8. http://dx.doi.org/10.35663/amp.2016.331.14.

11. Brasil. Ministério da Saúde. Secretaria de Vigilância em Saúde. Perspectivas brasileiras para o fim da tuberculose como problema de saúde pública. Bol Epidemiol. 2016;47(13):1-15.

12. Chirinos NEC, Meirelles BHS, Bousfield ABS. Relationship between the social representations of health professionals and people with tuberculosis and treatment abandonment. Texto Contexto Enferm. 2017;26(1):1-8. http://dx.doi.org/10.1590/0104-07072017005650015.

13. Organização Pan-Americana da Saúde. Direitos humanos, cidadania e tuberculose na perspectiva da legislação brasileira. Brasília: OPAS; 2015.

14. Soares MLM, Amaral NAC, Zacarias ACP, Ribeiro LKNP. Sociodemographic, clinical and epidemiological aspects of Tuberculosis treatment abandonment in Pernambuco, Brazil, 2001-2014. Epidemiol Serv Saude. 2017;26(2):369-78. http://dx.doi.org/10.5123/S1679-49742017000200014. PMid:28492778.

15. Brasil. Ministério da Saúde. Secretaria de Vigilância em Saúde. Departamento de Vigilância das Doenças Transmissíveis. Manual de Recomendações para o Controle da Tuberculose no Brasil. Brasília: Ministério da Saúde; 2019.

16. Brasil. Ministério da Saúde. Secretaria de Vigilância em Saúde. O controle da tuberculose no Brasil: avanços, inovações e desafios. Bol Epidemiol. 2014;44(1):1-13.

17. Heck MA, Costa JS, Nunes MF. Avaliação do programa de tuberculose em Sapucaia do Sul (RS): indicadores, 2000-2008. Cien Saude Colet. 2013;18(2):481-8. http://dx.doi.org/10.1590/S1413-81232013000200019. PMid:23358773.

18. Sapucaia do Sul. Secretaria Municipal de Saúde. Vigilância em saúde: plano municipal de controle da tuberculose. Sapucaia do Sul; 2005.

19. Heck MA, Costa JSD, Nunes MF. Prevalência de abandono do tratamento da tuberculose e fatores associados no município de Sapucaia do Sul (RS), Brasil, 2000-2008. Rev Bras Epidemiol. 2011;14(3):47885. http://dx.doi.org/10.1590/S1415-790X2011000300012. PMid:22069015.

20. Instituto Brasileiro de Geografia e Estatística. Ministério de Planejamento, Orçamento e Gestão. Centro de Documentação e Disseminação de Informações. Base de informações do censo demográfico 2010 : resultados do universo por setor censitário. Rio de Janeiro: IBGE; 2011.

21. Campoy LT, Arakawa T, Andrade RLP, Ruffino-Netto A, Monroe AA, Arcêncio RA. Quality and management of care to tuberculosis/hiv coinfection in the state of São Paulo, Brazil. Texto Contexto Enferm. 2019;28:e20180166. http://dx.doi.org/10.1590/1980-265x-tce-2018-0166.

22. Bastos $\mathrm{SH}$, Taminato M, Fernandes H, Figueiredo TMRM, Nichiata LYI, Hino P. Perfil sociodemográfico e de saúde da coinfecção tuberculose/HIV no Brasil: revisão sistemática. Rev Bras Enferm. 2019;72(5):1389-96. http://dx.doi.org/10.1590/0034-7167-2018-0285. PMid:31531666.

23. Gaspar RS, Nunes N, Nunes M, Rodrigues VP. Análise temporal dos casos notificados de tuberculose e de coinfecção tuberculose-HIV na população brasileira no período entre 2002 e 2012. J Bras Pneumol. 2016;42(6):416-22. http://dx.doi.org/10.1590/s1806-37562016000000054. PMid:28117471.

24. Chirinos NEC, Meirelles BHS. Fatores associados ao abandono do tratamento da tuberculose: uma revisão integrativa. Texto Contexto Enferm. 2011;20(3):599-606. http://dx.doi.org/10.1590/S010407072011000300023.

25. Heck MA, Costa JSD, Nunes MF. Prevalência de abandono do tratamento da tuberculose e fatores associados no município de Sapucaia do Sul (RS), Brasil, 2000-2008. Rev Bras Epidemiol. 2011;14(3):47885. http://dx.doi.org/10.1590/S1415-790X2011000300012. PMid:22069015.

26. Kigozi G, Heunis C, Chikobvu P, Botha S, van Rensburg D. Factors influencing treatment default among tuberculosis patients in a high burden province of South Africa. Int J Infect Dis. 2017;54:95-102. http:// dx.doi.org/10.1016/j.jij.2016.11.407. PMid:27894985. 
27. Furlan MCR, Oliveira SP, Marcon SS. Fatores associados ao abandono do tratamento de tuberculose no estado do Paraná. Acta Paul Enferm. 2012;25(spe1):108-14. http://dx.doi.org/10.1590/S010321002012000800017.

28. Viana PVS, Redner P, Ramos JP. Fatores associados ao abandono e ao óbito de casos de tuberculose drogarresistente (TBDR) atendidos em um centro de referência no Rio de Janeiro, Brasil. Cad Saude Publica. 2018;34(5):e00048217. http://dx.doi.org/10.1590/0102-311x00048217. PMid:29768580.

29. Santos TA, Martins MMF. Perfil dos casos de reingresso após abandono do tratamento da tuberculose em Salvador, Bahia, Brasil. Cad Saude Colet. 2018;26(3):233-40. http://dx.doi.org/10.1590/1414$462 \times 201800030235$

30. Silva PF, Moura GS, Caldas AJM. Fatores associados ao abandono do tratamento da tuberculose pulmonar no Maranhão, Brasil, no período de 2001 a 2010. Cad Saude Publica. 2014;30(8):1745-54. http://dx.doi. org/10.1590/0102-311X00124513. PMid:25210913.

31. Vieira AA, Leite DT, Adreoni S. Recorrência de tuberculose em município prioritário do estado de São Paulo. J Bras Pneumol. 2017;43(2):106-12. http://dx.doi.org/10.1590/s1806-37562016000000002. PMid:28538777.

32. Silva TC, Matsuoka PFS, Aquino DMC, Caldas AJM. Fatores associados ao retratamento da tuberculose nos municípios prioritários do Maranhão, Brasil. Cien Saude Colet. 2017;22(12):4095-104. http://dx.doi. org/10.1590/1413-812320172212.20612015. PMid:29267726.

33. Fregona G, Cosme LB, Moreira CMM, Bussular JL, Dettoni VV, Dalcolmo MP, et al. Risk factors associated with multidrug-resistant tuberculosis in Espírito Santo, Brazil. Rev Saude Publica. 2017;51(0):41. http:// dx.doi.org/10.1590/s1518-8787.2017051006688. PMid:28489185.

34. Silva SHA Jr, Mota JC, Silva RS, Campos MR, Schramm JMA. Descrição dos registros repetidos no Sistema de Informação de Agravos de Notificação, Brasil, 2008-2009. Epidemiol Serv Saude. 2016;25(3):487-98. http://dx.doi.org/10.5123/S1679-49742016000300005. PMid:27869920.

35. Brasil. Ministério da Saúde. Gabinete do Ministro. Secretaria de Vigilância em Saúde. Situação epidemiológica da tuberculose nos estados partes e associados do Mercosul 2009 a 2013. Brasília: Ministério da Saúde, 2015.

36. Sousa GJB, Garces TS, Pereira MLD, Moreira TMM, Silveira GM. Padrão temporal da cura, mortalidade e abandono do tratamento da tuberculose em capitais brasileiras. Rev Lat Am Enfermagem. 2019;27:e3218. http://dx.doi.org/10.1590/1518-8345.3019.3218. PMid:31826160.

37. Silva DR, Muñoz-Torrico M, Duarte R, GalvãoT, Bonini EH, Arbex FF, et al. Fatores de risco para tuberculose: diabetes, tabagismo, álcool e uso de outras drogas. J Bras Pneumol. 2018;44(2):145-52. http://dx.doi. org/10.1590/s1806-37562017000000443. PMid:29791552.

38. Peruhype RC, Acosta LMW, Ruffino A No, Oliveira MMC, Palha PF. The distribution of tuberculosis in Porto Alegre: analysis of the magnitude and tuberculosis-HIV coinfection. Rev Esc Enferm USP. 2014;48(6):1035 43. http://dx.doi.org/10.1590/S0080-623420140000700011. PMid:25626503.

39. Brasil. Ministério da Saúde. Secretaria de Vigilância em Saúde. Departamento de Vigilância Epidemiológica. Tratamento diretamente observado (TDO) da tuberculose na atenção básica: protocolo de enfermagem. Brasília: Ministério da Saúde; 2011.

40. Peruhype RC, Sicsú AN, Lima MCRAA, Hoffmann JF, Palha PF. Transferência de política: perspectiva do tratamento diretamente observado da tuberculose. Texto Contexto Enferm. 2018;27(3):e1710017. http:// dx.doi.org/10.1590/0104-070720180001710017.

41. Lavôr DCBS, Pinheiro JS, Gonçalves MJF. Avaliação da implantação da estratégia de tratamento diretamente observado para tuberculose em um município de grande porte. Rev Esc Enferm USP. 2016;50(2):247-54. http://dx.doi.org/10.1590/S0080-623420160000200010. PMid:27384204.

42. Clementino FS, Marcolino EC, Gomes LB, Guerreiro JV, Miranda FAN. Ações de controle da tuberculose: análise a partir do programa de melhoria do acesso e da qualidade da atenção básica. Texto Contexto Enferm. 2016;25(4):e4660015. http://dx.doi.org/10.1590/0104-07072016004660015.

43. Sá AMM, Santiago LA, Santos NVS, Monteiro NP, Pinto PHA, Lima AM, et al. Causas de abandono do tratamento entre portadores de tuberculose. Rev Soc Bras Clin Med. 2017;15(3):155-60. 